EPJ Web of Conferences 57, 04002 (2013)

DOI: $10.1051 /$ epjconf/20135704002

(C) Owned by the authors, published by EDP Sciences, 2013

\title{
Lightwave control of attosecond pulse emission from plasma mirrors
}

\author{
Antonin Borot ${ }^{1, a}$, Jonathan Wheeler ${ }^{2}$, Arnaud Malvache ${ }^{2}$, Sylvain Monchocé ${ }^{2}$, \\ Henri Vincenti ${ }^{2}$, Aurélien Ricci ${ }^{3}$, Fabien Quéré ${ }^{2}$ and Rodrigo Lopez-Martens ${ }^{2}$ \\ ${ }^{1}$ Laboratoire d'Optique Appliquée, ENSTA-Paristech, École Polytechnique, CNRS, \\ 91761 Palaiseau, France \\ ${ }^{2}$ Service des Photons, Atomes et Molécules, CEA, DSMIIRAMIS, CEN Saclay, \\ 91191 Gif-sur-Yvette, France \\ ${ }^{3}$ Laser Solutions Unit, Thales Optronique SA, 78990 Elancourt, France
}

\begin{abstract}
We demonstrate attosecond control of collective electron motion in plasmas driven by nearrelativistic intensity laser fields of controlled waveform in both space and time. We were able to generate spatially isolated attosecond pulses from a plasma mirrors for the first time.
\end{abstract}

\section{INTRODUCTION}

Today, waveform-controlled few-optical-cycle laser pulses are routinely used to launch and steer the motion of a few electrons in atoms, molecules and condensed matter with attosecond precision [1]. The aim of our group is to extend this control to the collective motion of electrons in plasmas using controlled light waveforms with relativistic intensity $\left(>10^{18} \mathrm{~W} . \mathrm{cm}^{-2}\right)$. In particular, the nonlinear distortion of relativistic lightwaves by plasma mirrors is a potential high-brightness source of attosecond light pulses in the extreme ultraviolet (EUV) spectral region $[2,3]$, which can be used to investigate a wide variety of ultrafast dynamical changes in matter [4-6].

\section{SUB-LIGHT-CYCLE ELECTRON DYNAMICS OF PLASMA MIRRORS}

When a femtosecond laser pulse is focused at intensities $>10^{16} \mathrm{~W} / \mathrm{cm}^{2}$ onto a solid, the atoms at the surface are instantaneously ionized by the leading edge of the pulse, thereby forming a thin layer of plasma with a sharp density gradient extending from zero in vacuum up to a few $10^{23}$ electrons. $\mathrm{cm}^{-3}$ over a few tens of nanometers. Where the electron density becomes over-critical (the "critical" density of a plasma is related to the laser frequency by the relation $n_{c}=m \epsilon_{0} \omega_{L}^{2} / e^{2} \approx 1.7 \times 10^{21} \mathrm{~cm}^{-3}$ at $800 \mathrm{~nm}$ ), the laser can no longer propagate into the plasma but instead is reflected by it, thereby creating a socalled plasma mirror [7].

At the plasma surface, the normal component of the intense oscillating laser electric field first launches the surface plasma electrons into vacuum and then steers them back into the density gradient [8] in the form of sub-cycle electron density peaks (see Fig 1b). Below the critical surface, the electronic density peak is freed of the grip of the laser field and propagates freely through the denser part of the plasma, where they trigger plasma waves oscillating at the local plasma frequency

\footnotetext{
ae-mail: antonin.borot@ensta-paristech.fr
}

This is an Open Access article distributed under the terms of the Creative Commons Attribution License 2.0, which permits unrestricted use, distribution, and reproduction in any medium, provided the original work is properly cited. 

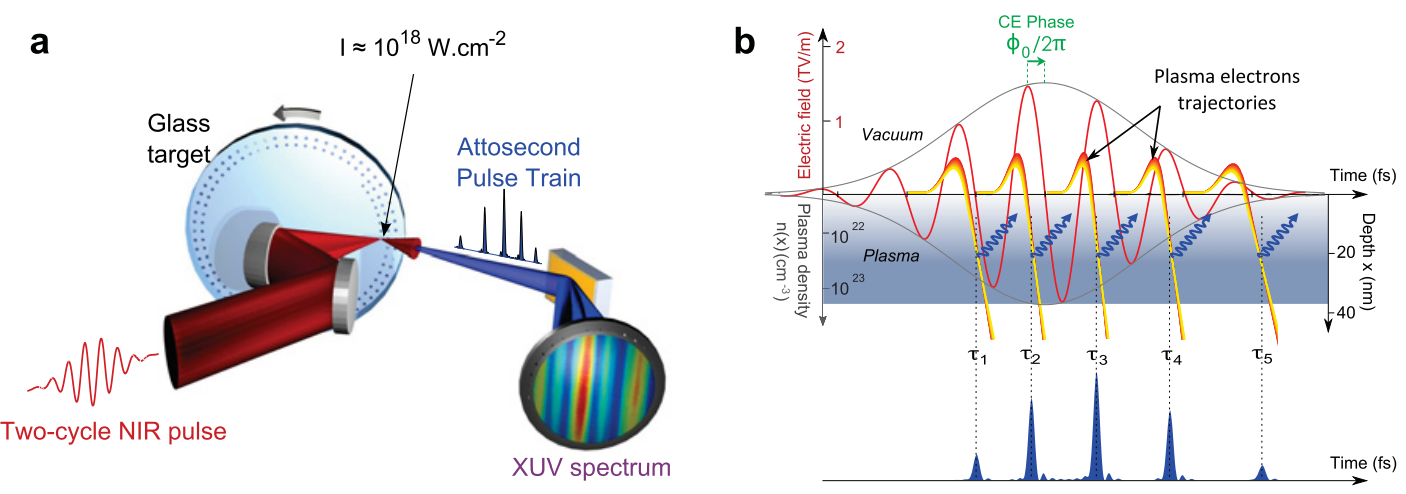

Figure 1. Sub-light-cycle electron dynamics of plasma mirrors. A two-cycle waveform controlled laser pulse is focused onto a solid glass target at near-relativistic intensity and generates a train of temporally-controlled attosecond pulse train, whose spectrum is recorded in a XUV spectrometer (a). At the plasma surface, the laser field (red) drives electron trajectories(yellow) into the overdense part of the plasma (grey) which emit an aperiodic attosecond pulse train (blue) (b).
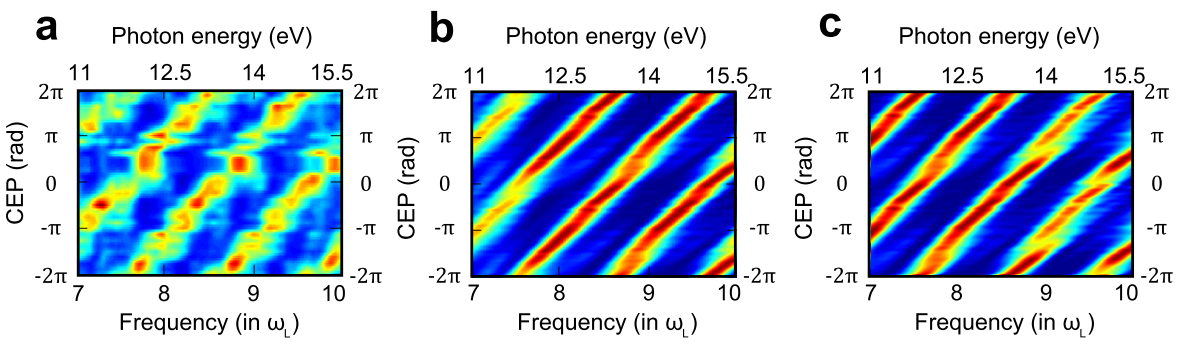

Figure 2. Evolution of XUV emission spectra with respect to the laser Carrier-Envelope Phase (CEP). Experimental (a), modeled (b) and simulation (c) results.

$\omega_{p}(x)=\sqrt{n(x) e^{2} / m \epsilon_{0}}$, where $n(x)$ is the local plasma density at depth $x$ in the gradient. Such longitudinal plasma waves can reemit transverse electromagnetic waves out of the plasma in the form of an attosecond burst of EUV radiation. As this process occurs once per laser optical cycle, it leads to the generation of an attosecond pulse train emitted in the direction of the reflected laser pulse $[9,10]$.

\section{LIGHTWAVE CONTROL OF ATTOSECOND PULSE GENERATION}

Because the collective electron trajectories are directly steered by the intense light waveform, the temporal structure of the attosecond pulse will map changes in the driving laser waveform. Through the level of control we can afford on the laser waveform, i.e. the drift in carrier-envelope phase (CEP) of the few-cycle laser pulses, we can expect a control of the plasma mirror dynamics with a sub-100 as precision.

In our experimental demonstration, we generate coherent XUV radiation from plasma mirrors driven on glass targets [11] by CEP-controlled $5 \mathrm{fs}$ (2-cycle) laser pulses [12]. Attosecond pulse trains are generated and their corresponding spectra of high-order laser harmonics are analyzed with an EUV spectrometer as a function of the laser CEP. Figure 2 shows the near-linear drift of the harmonic peaks in the spectra (integrated over 300 laser shots) as the CEP is swept through. The very strong dependence of the shape of the EUV spectrum with slight changes of the CEP $(250 \mathrm{mrad})$ proves that we can effectively control the plasma mirror dynamics with sub-200 as resolution [13]. 
a1

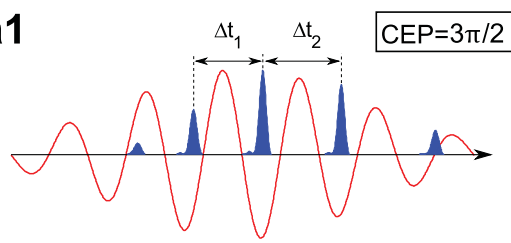

b1

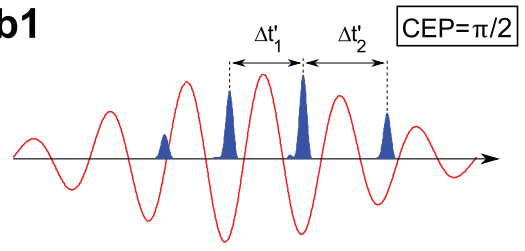

a2

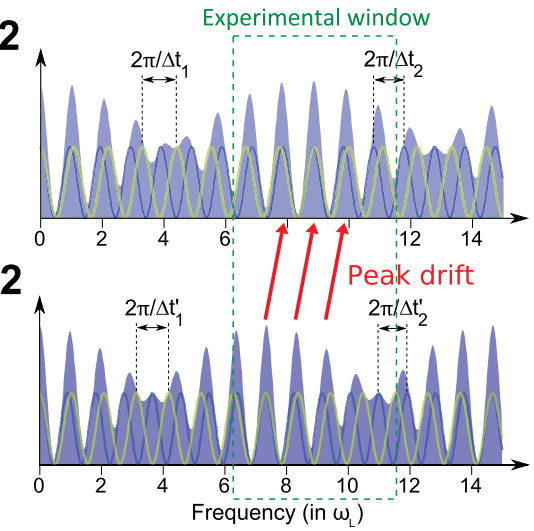

Figure 3. Spectral beating of the plasma emission. The aperiodic pulse train (a1) gives rise to beating in the spectral domain (a2) due to slight difference between the time separation between the three main attosecond pulse. When laser CEP is reduced, the attosecond time separation increases (b1), which induces a shift of the spectral pattern toward the lower frequencies (b2), which explains the drift of the spectral peaks with laser CEP.

In order to picture lightwave-driven plasma electron dynamics, we developed a simple onedimension model (see details in supplementary information of [13]) that solves the relativistic equation of motion of the electronic charges submitted to both laser and plasma electric fields and gives access to the temporal and spectral properties of the generated attosecond pulse train. The results of the model are in excellent agreement with the recorded CEP-dependent harmonic spectra (see Fig. 2b) as well as those generated by a comprehensive 2-dimensional "Particles In Cell" simulation (see Fig. 2c and supplementary information of [13]).

Our model highlights two key aspects of the attosecond dynamics of laser-driven plasma mirrors:

1. The fast time-variation of the few-cycle laser pulse envelope leads to an increasing temporal spacing between consecutive attosecond pulses along the pulse train (see Fig. 3a1). This leads to the disappearance of the typical "harmonic" spectral pattern in favor of a constructive spectral beating in the experimental detection window (see Fig. 3a2). This explains why the spectral peaks are separated approximately by the laser frequency but not located at harmonic orders of the laser frequency.

2. The temporal spacing between the consecutive pairs of attosecond pulses increases when the CEP is ramped upwards (see Fig. 3b1), shifting the region of constructive spectral interference towards higher frequencies, as shown in Fig. 3b2. This waveform-driven variation of the temporal structure of the attosecond pulse train explains the drift of the spectral peaks.

\section{ATTOSECOND LIGHTHOUSES: GENERATION OF A ISOLATED ATTOSECOND PULSES}

In this section, we show how spatial variation of the lightwave driving the plasma mirror can be controlled to isolate the individual attosecond pulses from the generated train.

Isolated attosecond pulses are more suited for most time-resolved imaging applications. We performed the first demonstration of a new technique called the "attosecond lighthouse effect" [14], which consists in introducing a rotation of the instantaneous laser wavefronts at focus by introducing angular dispersion in the beam prior to focusing (pulse-front tilt). If the wavefront rotation angle during the laser pulse is higher than the divergence of the high-harmonic emission, then each attosecond pulse of the train can be angularly separated after some propagation away from the plasma surface. Moreover, 
a

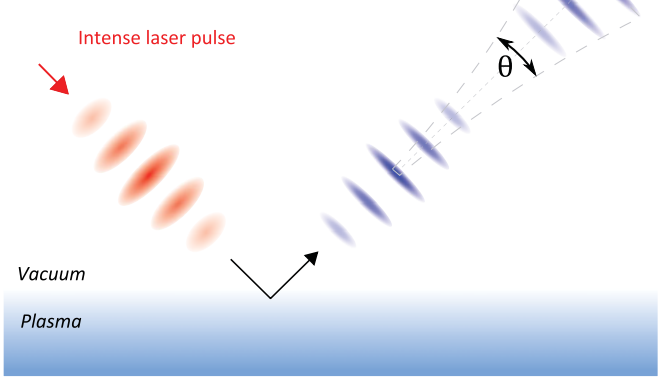

b

intense laser pulse with wave front rotation

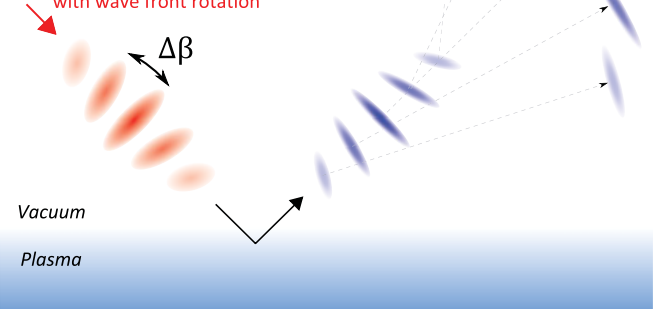

Figure 4. Principle of attosecond lighthouse from plasma mirror. The interaction between a laser pulse and a plasma mirror generate a train of attosecond pulses in the specular direction (a). By introducing wavefront rotation on the laser, each XUV pulse is generated in slight different direction, which lead after propagation to a bunch of spatially seperated isolated attosecond pulses (b).
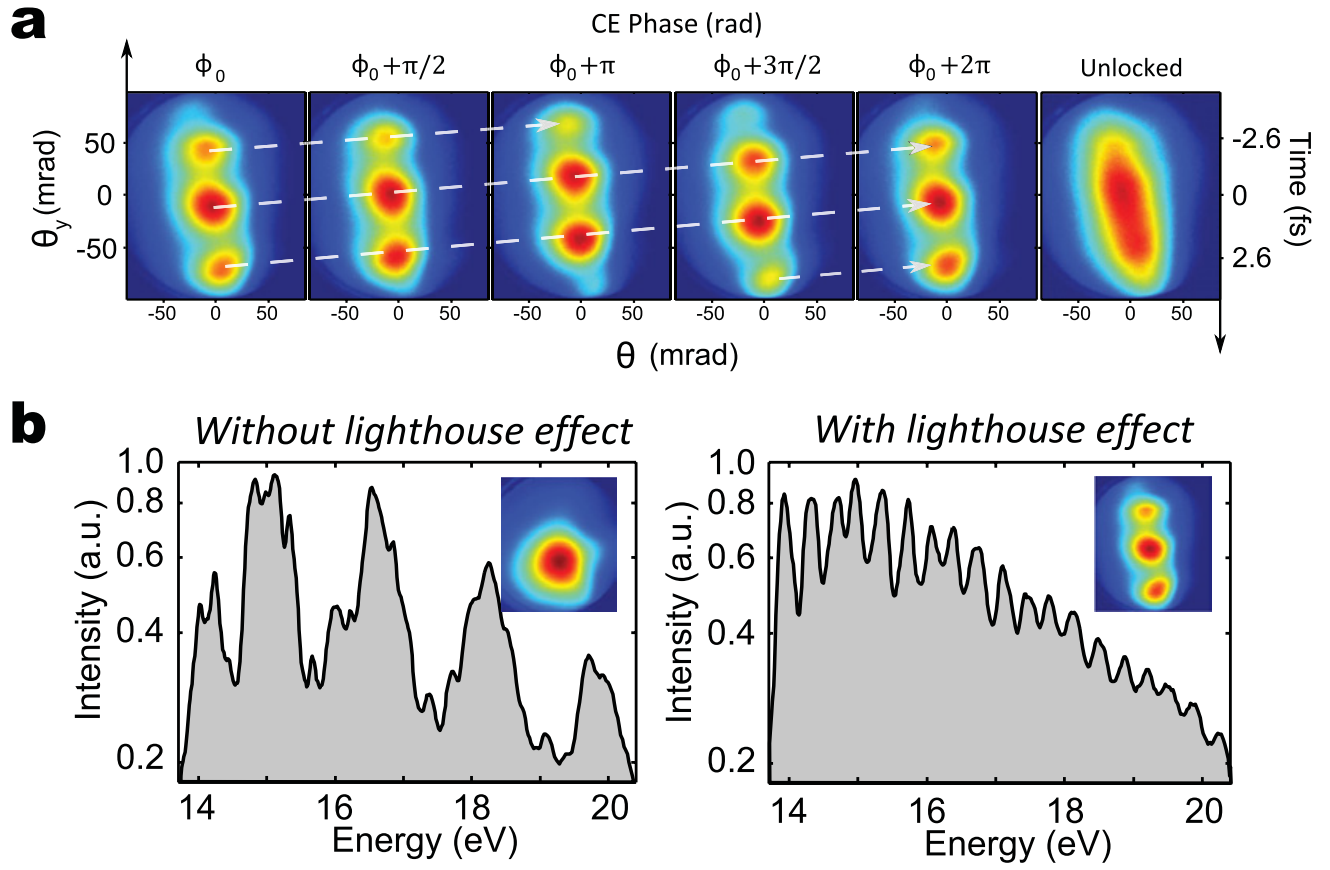

Figure 5. Experimental observation of the generation of isolated attosecond pulses. A group of three XUV beamlets is observed onto the spatial detector. By changing the laser CEP, the positions of the beamlets sweep along the detector with the same $2 \pi$-periodicity as the laser field. Intergration over 300 -unstabilized laser shots gives a blurry pattern (a). We also measured spectrum of the attosecond pulse train without wavefront rotation (harmonic-like spetrum) and the spectrum of an idividual XUV beamlet with wavefront rotation, which exhibits a disappearance of the $\omega_{L}$-oscillation, characteristic of an isolated attosecond pulse (b). 
the direction of emission of each individual attosecond pulse can be controlled by simply changing the laser CEP.

One important aspect of the lighthouse effect is its simplicity in the case of few-cycle pulses. Indeed, the wavefront rotation velocity at focus can be controlled by controlling the tilt of a glass wedge placed in the laser beam prior to focusing. A spatial EUV detector placed sufficiently far down the path of the reflected laser beam can be used to resolve spatial separation of the individual attosecond light beams.

Figure 5a shows the spatial EUV emission profile as a function of wavefront rotation velocity. We observe several angularly separated beamlets on the detector, each corresponding to an individual attosecond pulse in the train. To confirm this, we continuously vary the CEP and observe, as expected, the relative change in the amplitude and direction of individual attosecond pulse emission with the same $2 \pi$-periodicity as the oscillating laser field itself. Finally, we measured the spectrum of the emission at the center of the EUV beam, with and without lighthouse effect (see Fig. 5b) and observed a transition from a modulated harmonic-like spectrum, typically associated to a train of co-propagating attosecond pulses, into a continuous spectrum, characteristic of an isolated attosecond pulse. This is the first demonstration of single attosecond pulse generation from plasma mirrors driven at near-relativistic intensity [15].

\section{CONCLUSION}

In this paper, we describe recent experimental milestones in our quest to control relativistic plasma mirror dynamics on the attosecond time scale. We show how controlling the temporal waveform of the driving few-cycle laser field with near-relativistic intensity can control the relative timing of attosecond pulse train emitted by the plasma mirror. We also show how this can be coupled to controlling the spatial evolution of the laser waveform in order to isolate individual attosecond pulses from the train. When driven at higher intensities, this process should lead to the production of bright attosecond EUV pulses for numerous applications.

\section{References}

[1] A. Baltuska, T. Udem, M. Uiberacker, M. Hentschel, E. Goulielmakis, C. Gohle, R. Holzwarth, V. S. Yakovlev, A. Scrinzi, T. W. Hansch, and F. Krausz, Nature 421, 611 (2003).

[2] G. Tsakiris, K. Eidmann, J. M. ter Vehn, and F. Krausz, New Journal of Physics 8 (2006).

[3] N. M. Naumova, J. A. Nees, I. V. Sokolov, B. Hou, and G. A. Mourou, Phys. Rev. Lett. 92, 063902 (2004).

[4] M. Uiberacker, T. Uphues, M. Schultze, A. J. Verhoef, V. Yakovlev, M. F. Kling, J. Rauschenberger, N. M. Kabachnik, H. Schroder, M. Lezius, K. L. Kompa, H.-G. Muller, M. J. J. Vrakking, S. Hendel, U. Kleineberg, U. Heinzmann, M. Drescher, and F. Krausz, Nature 446, 627 (2007).

[5] M. Drescher, M. Hentschel, R. Kienberger, M. Uiberacker, V. Yakovlev, A. Scrinzi, T. Westerwalbesloh, U. Kleineberg, U. Heinzmann, and F. Krausz, Nature 419, 803 (2002).

[6] A. L. Cavalieri, N. Muller, T. Uphues, V. S. Yakovlev, A. Baltuska, B. Horvath, B. Schmidt, L. Blumel, R. Holzwarth, S. Hendel, M. Drescher, U. Kleineberg, P. M. Echenique, R. Kienberger, F. Krausz, and U. Heinzmann, Nature 449, 1029 (2007).

[7] W. L. Kruer, The Physics of Laser Plasma Interation (1988).

[8] F. Brunel, Phys. Rev. Lett. 59, 52 (1987).

[9] F. Quere, C. Thaury, P. Monot, S. Dobosz, P. Martin, J.-P. Geindre, and P. Audebert, Physical Review Letters 96, 125004 (2006).

[10] Y. Nomura, R. Horlein, P. Tzallas, B. Dromey, S. Rykovanov, Z. Major, J. Osterhoff, S. Karsch, L. Veisz, M. Zepf, D. Charalambidis, F. Krausz, and G. D. Tsakiris, Nat Phys 5, 124 (2009). 


\section{EPJ Web of Conferences}

[11] A. Borot, A. Malvache, X. Chen, D. Douillet, G. Iaquianiello, T. Lefrou, P. Audebert, J.-P. Geindre, G. Mourou, F. Quéré, and R. Lopez-Martens, Opt. Lett. 36, 1461 (2011).

[12] X. Chen, A. Malvache, A. Ricci, A. Jullien, and R. Lopez-Martens, Laser Physics pp. 1-4 (2010).

[13] A. Borot, A. Malvache, X. Chen, A. Jullien, J.-P. Geindre, P. Audebert, G. Mourou, F. Quere, and R. Lopez-Martens, Nat Phys 8, 416 (2012).

[14] H. Vincenti and F. Quéré, Phys. Rev. Lett. 108, 113904 (2012).

[15] J. A. Wheeler, A. Borot, S. Monchoce, H. Vincenti, A. Ricci, A. Malvache, R. Lopez-Martens, and F. Quere, Nat Photon 6, 829 (2012). 